\title{
Is your ischemic patient an ISCHEMIA patient?
}

\author{
Sean R. McMahon, $M D,{ }^{a}$ and W. Lane Duvall, $M D^{a}$ \\ a Heart and Vascular Institute, Hartford Hospital, Hartford, CT
}

Received Jun 22, 2021; accepted Jun 24, 2021

doi: $10.1007 / \mathrm{s} 12350-021-02726-3$

\section{See related article, pp. 1100-1105}

The ISCHEMIA trial (International Study of Comparative Health Effectiveness with Medical and Invasive Approaches) published last year adds to decades of literature regarding patient selection for coronary revascularization. ${ }^{1}$ While it is generally agreed upon that patients presenting with acute coronary syndromes should receive revascularization, the superiority of medical therapy versus revascularization in patients with stable angina remains a topic of debate. While previous retrospective literature suggested that patients with $>10$ $12.5 \%$ ischemia on SPECT myocardial perfusion imaging who underwent revascularization had lower mortality than those who did not, ${ }^{2,3}$ the ISCHEMIA trial sought to add to the literature by prospectively assessing a patient population with a high ischemic burden. However, while this may have been the goal, it might not have been what was accomplished.

The ISCHEMIA trial was a multicenter randomized controlled trial of 5,179 patients with stable coronary artery disease with moderate-to-severe ischemia comparing medical therapy versus medical therapy and revascularization. All enrolled patients had a clinically indicated stress test demonstrating moderate or severe reversible ischemia by nuclear imaging $(\geq 3 / 16$ segments), cardiac magnetic resonance imaging ( $>12 \%$ or $\geq 3 / 16$ segments), echocardiography ( $\geq 3 / 16$ segments), or high-risk findings on exercise tolerance testing. Those with recent acute coronary syndrome, renal impairment with a glomerular filtration rate of $<30 \mathrm{ml} / 1.73 \mathrm{~m} 2$ BSA, left main coronary artery stenosis, ejection fraction of less than 35\%, New York Heart Association class III or IV heart failure symptoms, or uncontrolled angina

Reprint requests: W. Lane Duvall, MD, Heart and Vascular Institute, Hartford Hospital, Hartford, CT ; Lane.Duvall@hhchealth.org J Nucl Cardiol 2022;29:1106-8.

$1071-3581 / \$ 34.00$

Copyright (C) 2021 American Society of Nuclear Cardiology. despite medical therapy were excluded. These patients were randomized to optimal medical therapy plus early revascularization or medical management alone. Coronary computed tomography angiography was used to exclude left main disease when feasible. At a median follow-up of 3.2 years, there was no significant difference between the groups regarding the primary outcome of a composite of cardiovascular death, myocardial infarction, or hospitalization for heart failure, unstable angina, or cardiac arrest. However, a notable difference between the groups was the reduction in anginal symptoms among patients in the invasive strategy group. Of those in the medical therapy group $15 \%$ crossed over to receive revascularization before a primary endpoint was reached and an additional $6 \%$ underwent PCI after the occurrence of a primary event. Among patients fitting the ISCHEMIA inclusion and exclusion criteria, this trial supports optimal medical therapy with an invasive strategy reserved for those with uncontrolled angina.

The ISCHEMIA trial had its roots in the COURAGE trial which randomized 2,287 patients with significant coronary artery disease to percutaneous coronary intervention with optimal medical therapy or medical therapy alone. ${ }^{4}$ After 4.6 years of follow-up, no benefit of revascularization over optimal medical therapy for patients with stable coronary artery disease was found in regards to the primary endpoint of death and nonfatal myocardial infarction. A particular concern of the COURAGE trial was an under representation of patients with moderate or severe ischemia who were thought to be the most likely to benefit from revascularization. Accordingly, investigators analyzed a subset of patients in the COURAGE trial who had serial myocardial perfusion imaging and found a greater reduction in ischemic myocardium in patients who underwent revascularization. ${ }^{5}$ Although not powered as a clinical outcomes trial, patients in this subset of the COURAGE trial who experienced a reduction in ischemic burden had a lower rate of death or nonfatal MI (13.4\% vs $24.7 \%)$. These results suggested that patients with a larger ischemic burden may benefit from 
revascularization, the primary clinical question sought to be addressed by the ISCHEMIA trial.

Unfortunately, the ISCHEMIA patient population initially sought by investigators would never be brought to fruition. Poor enrollment and the need to facilitate recruitment fundamentally changed the patient population studied in ISCHEMIA through a series of protocol modifications. ${ }^{6}$ The requirement for core lab confirmation of the ischemic burden prior to randomization and eventually even the need for imaging assessment of ischemia were eliminated in the name of jump-starting stalled enrollment. This resulted in $24.5 \%$ of the trial population being enrolled based on stress ECG alone. And odder still, these non-imaging patients were characterized as having "severe" ischemia which likely resulted in the unusual result of patients with severe ischemia having a better prognosis than those with moderate ischemia $(12.7 \%$ vs $13.8 \%$ event rate at follow-up). Further moving the patient population away from one with moderate-to-severe ischemia was the protocol modification allowing for lower amounts of ischemia if a patient's exercise capacity was less than 7 METs. In the end, only $33.8 \%$ of the study population had imaging evidence for severe myocardial ischemia (and not all confirmed by the core lab) and only $18.7 \%$ had severe ischemia on nuclear imaging. Other confounders to the study's goal of assessing patients with significant ischemia and its general applicability were the high proportion of patients (approximately 35\%) who were asymptomatic, the small (only 10\%) proportion of patients with perfusion studies performed in the USA, as well as the varying referral patterns and patient care found outside of the USA where a majority of patients were enrolled.

And that takes us to this issue of the Journal, wherein Al-Mallah and colleagues sought to add to our understanding of the prognosis of patients with moderate-to-severe ischemia seen at their institution (Houston Methodist Hospital - a large tertiary care hospital) and how these patients compared with the patients enrolled in the ISCHEMIA trial. ${ }^{7}$ The authors were concerned about the generalizability of the ISCHEMIA trial results to real-world practice given that of the nearly 26,000 patients who underwent screening, less than half $(47 \%)$ met the inclusion criteria, and only a fifth were ultimately randomized. This was in part due to the broad exclusion criteria including ejection fraction $<30 \%$, NYHA class III-IV heart failure, unacceptable angina despite maximal medical therapy, and left main stenosis. In a retrospective analysis, the authors reviewed their patients with moderate-to-severe ischemia $(>10 \%$ ischemia) over a 3 -year period of time to see who would have been eligible for the ISCHEMIA trail.
Over 14,000 patients were retrospectively assessed and 1,534 had a perfusion defect (only 10.7\%). Of these, 709 had moderate-to-severe ischemia, but $66 \%$ had at least one exclusion criteria for the ISCHEMIA trial. This is an impressive reduction in number (from 14,283 down to just 230) in itself and raises further concerns about the generalizability of the ISCHEMIA trial. So how do those left out fare? All-cause mortality was found to be significantly higher in those with moderate-to-severe ischemia who would have been excluded from the trial $(11.3 \%$ vs $3.9 \%, \mathrm{p}<0.001)$. This adds to the concern about the generalizability of the ISCHEMIA trial and poses the question of how to manage those not included in the study. While this study by Al-Mallah et al cannot answer this question, and while it is limited by the lack of cardiac-specific endpoints as well as details of the medical therapy, it emphasizes the nature of the lowerrisk cohort of moderate-to-severe ischemia patients included in ISCHEMIA. Because of this, careful consideration should be given to the management of the patient with moderate-to-severe ischemia on perfusion imaging seen in clinical practice as it is not clear that revascularization would not be beneficial for these patients with ISCHEMIA exclusion criteria.

This paper is likely the first in a series of secondary analyses (unofficial or official) of the ISCHEMIA trial that we will see. ISCHEMIA in its final form was not a trial evaluating revascularization versus medical therapy in patients with moderate-to-severe ischemia $(>10 \%)$ due to significant mid-study protocol changes, nor was it a trial of coronary CTA versus myocardial perfusion imaging as some would suggest (a topic for another time). Al-Mallah and colleagues remind us that many common patients that we see in clinical practice with significant ischemia were excluded from the ISCHEMIA trial and the mortality of these patients is higher than those included. These patients, and perhaps even patients with moderate-to-severe ischemia by imaging, do not have iron clad clinical trial evidence to guide their care, but instead we must continue to rely on good clinical judgment which puts the patient first, something that nuclear cardiology has excelled in doing for decades.

\section{References}

1. Maron DJ, Hochman JS, Reynolds HR, Bangalore S, O'Brien SM, Boden WE et al. Initial Invasive or Conservative Strategy for Stable Coronary Disease. N Engl J Med 2020;382:1395-407.

2. Hachamovitch R, Hayes SW, Friedman JD, Cohen I, Berman DS. Comparison of the short-term survival benefit associated with revascularization compared with medical therapy in patients with no prior coronary artery disease undergoing stress myocardial 
perfusion single photon emission computed tomography. Circulation 2003;107:2900-7.

3. Hachamovitch R, Rozanski A, Shaw LJ, Stone GW, Thomson LE, Friedman JD et al. Impact of ischaemia and scar on the therapeutic benefit derived from myocardial revascularization vs. medical therapy among patients undergoing stress-rest myocardial perfusion scintigraphy. Eur Heart J 2011;32:1012-24.

4. Boden WE, O'Rourke RA, Teo KK, Hartigan PM, Maron DJ, Kostuk WJ et al. Optimal medical therapy with or without PCI for stable coronary disease. N Engl J Med 2007;356:1503-16.

5. Shaw LJ, Berman DS, Maron DJ, Mancini GB, Hayes SW, Hartigan PM et al. Optimal medical therapy with or without percutaneous coronary intervention to reduce ischemic burden: results from the
Clinical Outcomes Utilizing Revascularization and Aggressive Drug Evaluation (COURAGE) trial nuclear substudy. Circulation 2008;117:1283-91.

6. Murthy VL, Bateman TM, Chen W, Malhotra S, Miller EJ, Ruddy TD et al. Impact of the ISCHEMIA Trial on Stress Nuclear Myocardial Perfusion Imaging. J Nucl Med 2020;61:962-4.

7. Al-Mallah M, Ahmed AI, Nabi F, Chang SM, Kleiman N, ChamsiPasha $\mathrm{M}$ et al. Outcomes of patients with moderate to severe ischemia excluded from the ischemia trial. J Nuc Cardiol 2021.

Publisher's Note Springer Nature remains neutral with regard to jurisdictional claims in published maps and institutional affiliations. 